\title{
The heterogeneity and dynamic equilibrium of rat embryonic stem cells
}

Cell Research (2011) 21:1143-1147. doi:10.1038/cr.2011.98; published online 14 June 2011

\section{Dear Editor,}

Embryonic stem cells (ESCs) are pluripotent cells established from early stage embryos that maintain the ability to differentiate into three-germ layer cells. This unique characteristic is the basis for various applications of ESC technology. To successfully use ESCs in these applications, researchers have explored a variety of in vitro methods to maintain the self-perpetuating, naïve, undifferentiated state during long-term culture of ESCs. The first ESCs were established from the inner cell mass (ICM) of mouse blastocysts in 1981 [1, 2]. Initially, mouse ESCs (mESCs) were derived and cultured on layers of mitotically inactivated fibroblasts in serumcontaining medium $[1,2]$. mESCs require a combination of leukemia inhibitory factor (LIF) $[3,4]$ and bone morphogenetic protein 4 (BMP4) to maintain their selfrenewal state [5]. Recently, culture medium based on a combination of two small molecule inhibitors has been demonstrated to support self-renewal [6]. PD0325901 and CHIR99021 inhibit the mitogen-activated protein/ extracellular signal-regulated kinase (ERK) kinase (MEK) and glycogen synthase kinase 3 (GSK3), respectively. This combination of inhibitors is termed ' $2 \mathrm{i}$ '. Recent breakthroughs have been made in establishing and culturing rat ESCs (rESCs) from the ICM of rat embryos under molecularly defined conditions with $2 \mathrm{i}$ plus LIF (2i-LIF) to maintain a pluripotent state [7, 8]. These rESCs resemble mouse ESCs in that they form compact and domed clones, can be extensively expanded by single cell passage and use similar signaling pathways for self-renewal $[7,8]$. Thus, undifferentiated rESCs are generally regarded as a stable population of cells with a low level of background differentiation under $2 \mathrm{i}$ culture conditions. Here, we report heterogeneity of rESCs under standard 2i-LIF culture conditions.

Initially, preimplantation blastocysts (embryonic day 4.5) from an outbred strain of CD:VRL1 rats (also called Sprague-Dawley (SD)) and inbred strains of Fischer 344 and Brown-Norway $(\mathrm{BN})$ rats were individually seeded onto mitomycin C-treated mouse embryonic fibroblast cells in N2B27 medium supplemented with 2i-LIF (Supplementary information, Data S1, Figure 1A and 1B). The attached embryos robustly generated outgrowths containing Oct4-positive cells, indicating the presence of ES progenitor cells (Supplementary information, Data S1, Figure 1C). After mechanical fragmentation of the outgrowths into small clumps and replating on fresh feeder cell layers, ES-like clones with a compact, domed morphology emerged. Surprisingly, after dissociation of these ES-like clones into single cells and seeding them onto fresh feeders, two distinct clone morphologies appeared (Figure 1B). One population consisted of domed, round, tightly packed colonies termed "d-rESCs." The other population took on the appearance of flattened clones termed flattened rat ESCs (f-rESCs). The latter were similar in appearance to mouse epiblast stem cells (Figure 1B). To determine whether the specific culture condition affects the number of d-rESCs or f-rESCs, we cultured r-ESCs in $2 \mathrm{i}$ medium without LIF. However, we found that the pluripotent state of rESCs colonies could not be maintained upon the removal of LIF. In addition, we have tried the concentration of PD0325901 from 0.4 to $1 \mu \mathrm{M}$, and found that the ratio between flat colonies and dome colonies is similar and the dome colonies could not be further enriched. We also found that when rESCs were cultured in the medium with 2-3 $\mu \mathrm{M}$ PD0325901, they proliferated poorly and could not be maintained beyond passage 4 (data not shown).

We found that these f-rESCs and d-rESCs subpopulation could be separated by mechanical method. The d-rESCs colonies attached less well to feeder layers than f-rESCs colonies did. To distinguish and collect two morphologically distinct cell populations for functional study in our work, we suspended the dome-shaped colonies by gently pipetting up and down under a stereomicroscope. Then the suspended d-rESCs were split and replated on a new dish. On the other hand, the f-rESCs colonies were picked up by mechanical microdissection under a stereomicroscope and replated to a new culture dish. Both cell lines were extensively propagated from single cells with normal karyotypes and were passaged 
at 3-day intervals (Supplementary information, Data S1, Figure S1B). Interestingly, after dissociation into single cells and seeding onto fresh feeder cells, the domed cells rarely formed flat clones, whereas the flat cells formed both domed and flat clones. To explore the culture conditions distinguishing these two kinds of cells, we tried different passage protocols, seeding density, feeder cell densities and growth factors. Seeding density affected clone morphology. When the flat cells were seeded at high density (approximately $5 \times 10^{4}$ cells $/ \mathrm{cm}^{2}$ ), more than $95 \%$ of the cells were flat, whereas at low density (about $2.5 \times 10^{4}$ cells $/ \mathrm{cm}^{2}$ ), half of the clones showed a domed morphology.

To investigate whether the domed colonies and the flattened colonies were both pluripotent, we measured their expression of pluripotent markers. Even after 30 passages, both cell types showed positive staining for alkaline phosphatase and expressed the pluripotency markers Oct4, Nanog, Sox2 and SSEA1 (Supplementary information, Data S1, Figures S1A and S2A). To further confirm the pluripotency of these cells, we differentiated them by embryoid body formation (in vitro) (Supplementary information, Data S1) and teratoma formation (in vivo) (Supplementary information, Data S1). After differentiating the cells in vitro, the resulting cells were positive for markers of all three germ layers (Supplementary information, Data S1, Figure S2B). In addition, histological examination of teratomas harvested 6 weeks after injection under the skin of NOD-SCID mice showed that both cell types gave rise to various tissues of all three germ layers (Supplementary information, Data S1, Figure S2C). Furthermore, both domed and flattened cell colonies from BN rats contributed to chimeras (Supplementary information, Data S1, Figure S2D and S2E). Chimeric rats were also obtained after electroporation of the pCAG-EGFP plasmid into the domed cell line from CD:VRL1 rats (Supplementary information, Data S1, Figure S1C and S1D). These results demonstrate that both of these morphologically distinct cell populations retain pluripotent characteristics.

To investigate the molecular properties of d-rESCs and f-rESCs, a global analysis of gene expression was performed using the Affymetrix rat expression array 230 2.0. A total of 213 transcripts having the gene symbol were specifically enriched in f-rESCs. These transcripts were upregulated more than 2-fold in f-rESCs compared to d-rESCs, representing a significant difference (Affymetrix "P" call) (Supplementary information, Data S1, Table S1). Figure 1E lists 16 genes whose RNA expression levels were significantly altered in f-rESCs. The f-rESCs expressed many molecular marker genes at high levels, including endodermal markers (Gata6 and Sox17), a lymphocyte marker (CD81), a hepatocyte surface marker (Hes6) and a mesenchymal marker (Vimentin) (Supplementary information, Table S2). On the other hand, some markers of pluripotent cells, including Pecam 1, Tbx3, Cdh1 (E-cadherin), Gbx2 and Sox2, did not show any difference between f-rESCs and d-rESCs. In fact, cluster analysis of f-rESCs using a database showed that they were not similar to d-rESCs (Supplementary information, Figure S3).

Signal transduction is essential for ESC derivation. The fundamental obstacles involved in the generation of ESCs include spontaneous apoptosis, spontaneous differentiation and cell-cycle arrest in "nonpermissive" species. The MAPK signaling pathway is known to play an essential role in autoinductive differentiation and we detected enrichment of genes concentrated in MAPK signaling in f-rESCs (Supplementary information, Table $\mathrm{S} 1)$. The downstream targets that respond rapidly to ERK-mediated activation of transcription factors are enriched in f-rESCs such as Fos, Egrl and Jun (Figure 1E). ESCs have a unique cell cycle that is thought to underlie the short G1 and rapid entry of ES cells into S-phase. Upon differentiation the G1 phase becomes longer. We discovered that genes that are implicated in cell cycle arrest were enriched in f-rESCs, including Gadd45a, Gadd45b, Gadd45g and Cdkn1a (P21). Conversely, cyclin $\mathrm{B} 1$ was highly expressed in d-rESCs. Changes in the p53 pathway also affect pluripotency and cellular dedifferentiation. In our microarray data, genes in the p53 pathway were enriched in f-rESCs, including Gadd45a (Ddit1), Cdknla (P21), Perp and Tp53inpl (Supplementary information, Table $\mathrm{S} 1)$.

To further investigate whether the genetic background between the f-rESCs and d-rESCs was different, we compared the gene expression patterns of both cell types by real-time PCR. First, expression of genes known to be enriched in conventional ESCs such as Stella, Oct4, Sox2, Nanog and Klf4 was detected (Figure 1D, Supplementary information, Data S1). The f-rESCs expressed similar levels of Oct4 and Sox2 compared to d-rESCs, but higher levels of Nanog, Klf4 and Stella (Figure 1D). We then compared expression levels of the EpiSC-enriched genes Gata6, Sox17, Cerl and Fgf5. Fgf5 was not detected in d-rESCs or f-rESCs cells (data not shown); however, the other genes evaluated (Gata6 and Sox 17) were highly expressed in f-rESCs (Figure 1D). f-rESCs expressed Cerl at similar levels compared with d-rESCs (Figure 1D). As the d-rESCs attached less well to feeder layers than f-rESCs cells did, we compared the expression of integrins, which play a role in cell adhesion. All of the examined integrins were clearly expressed at higher levels in f-rESCs than in d-rESCs 
A

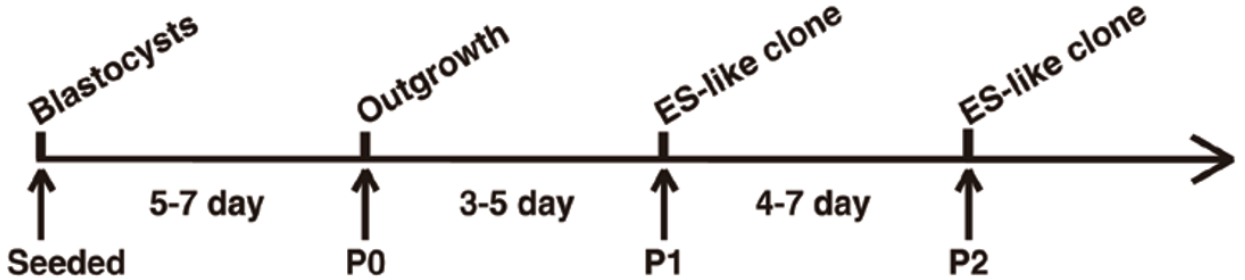

B
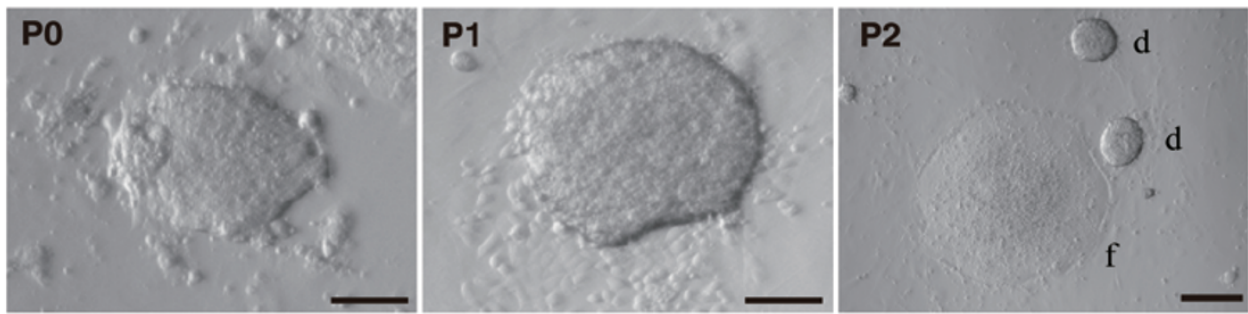

C
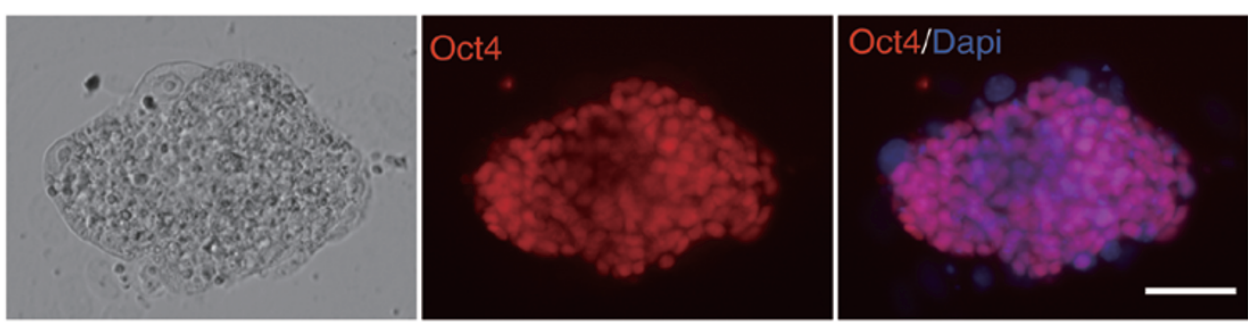

D

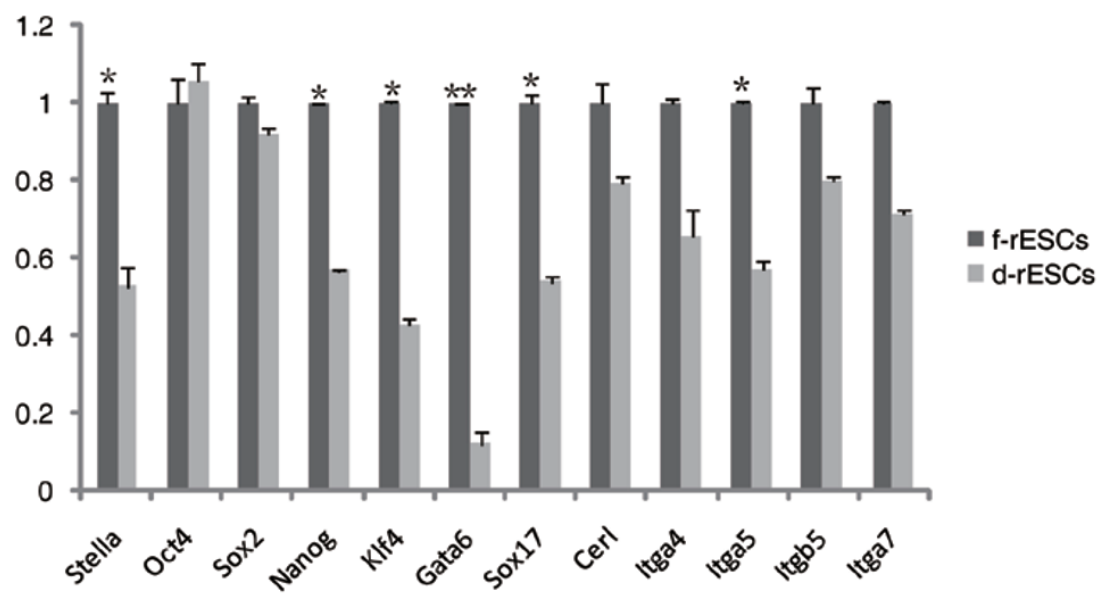

E

\begin{tabular}{|c|c|c|c|}
\hline Gene Title & $\begin{array}{l}\text { Gene } \\
\text { Symbol }\end{array}$ & UniGene & $\begin{array}{l}\text { RefSeq } \\
\text { Transcript }\end{array}$ \\
\hline FBJ osteosarcoma oncogene & Fos & Rn. 103750 & NM_022197 \\
\hline Kruppel-like factor 15 & Klf15 & Rn. 22556 & NM_053536 \\
\hline early growth response 1 & Egr1 & Rn. 9096 & NM_012551 \\
\hline basic helix-loop-helix domain containing, class B3 & Bhlhb3 & Rn. 10784 & NM_133303 \\
\hline vimentin & Vim & Rn. 2710 & NM_031140 \\
\hline dual specificity phosphatase 9 & Dusp9 & Rn. 100548 & NM_001037973 \\
\hline PHD finger protein 17 & Phf 17 & Rn. 90858 & NM_001107670 \\
\hline prostaglandin E synthase & Ptges & Rn. 7730 & NM_021583 \\
\hline Interferon regulatory factor 8 & $\operatorname{Irf} 8$ & Rn. 3765 & NM_001008722 \\
\hline nuclear RNA export factor 7 & Nxf7 & Rn. 24735 & NM_001037216 \\
\hline synaptonemal complex protein 3 & Sycp3 & Rn. 34889 & NM_013041 \\
\hline Jun oncogene & Jun & Rn. 93714 & NM_021835 \\
\hline growth arrest specific 6 & Gas6 & Rn. 52228 & NM_057100 \\
\hline ADAMTS-like 4 & Adamts14 & Rn. 62115 & NM_001034012 \\
\hline growth arrest and DNA-damage-ind & Gadd $45 \mathrm{~g}$ & Rn. 16950 & NM_001077640 \\
\hline growth arrest and DNA-damage-induc & Gadd $45 b$ & Rn. 35886 & NM_001008321 \\
\hline
\end{tabular}


Figure 1 (A) Scheme of the derivation of embryonic stem cells. Blastocysts on embryonic day 4.5 were seeded individually on MEF feeders. After 5-7 days, outgrowths were passaged by mechanically cutting them into small pieces and seeding them onto fresh MEF feeders, recorded as passage 0 (P0). After another 3-5 days, ES-like clones were passaged by sequential digestion into single cells and plated on fresh MEF feeders (P1). ES-like clones were then further passaged with Accutase. (B) Phase contrast image of ES-like clones at P0, P1 and P2. ES-like clones with two kinds of morphologies, flat (f) and domed (d), were observed at P2. Bar $=50 \mu \mathrm{m}$. (C) Immunofluorescence staining for Oct4 in day 5 outgrowths. Bar $=50 \mu \mathrm{m}$. (D) Expression of mES-enriched genes (stella, oct4, nanog, sox2 and klf4), mEpiSC-enriched genes (gata6, sox17 and cerl1) and integrin-related genes (Itga4, Itga5, Itgb5 and Itga7) in f-rESCs and d-rESCs. Three biological replicates were used for both kinds of cells. The relative expression of each gene in f-rESCs and d-rESCs was normalized to its expression in f-rESCs after normalization to Gapdh. Statistically significant $P$ values are noted with an asterisk $(n=3)$. ${ }^{*} P<0.05$ and ${ }^{* *} P<0.001$. (E) Enriched genes expressed at more than 5-fold higher levels in f-rESCs.

(Figure 1D). The gene expression patterns of f-rESCs did not resemble those reported for conventional mESCs or for EpiSCs, indicating that some subpopulations of f-rESCs might have a unique pattern of gene expression. Though the gene expression level of Oct4 and Sox2 is similar between f-rESCs and d-rESCs, the expression level of Gata6 or Sox 17 was higher in the cell population of f-rESCs comparing to d-rESCs by both q-PCR and microarray analyses. This indicated that f-rESCs were unstable and some cells in the f-rESCs might be primed to differentiate.

In summary, here, we report for the first time the heterogeneity of pluripotent rESCs in the same culture environment. Two rESCs subpopulations could be separated by mechanical method. Our study revealed two distinct pluripotent cell subpopulations, d-rESCs and f-rESCs, within ESC populations derived from different rat species, including SD, Fischer 344 and $\mathrm{BN}$ rats, using chemically defined culture medium with 2 i-LIF. Critical differences, however, were also found between these two subpopulations. Our results indicate that different expression levels of regulators within the MAPK and p53 pathways might be related to the heterogeneous characteristics of rESCs under 2i-Lif conditions. The distinct gene expression patterns in f-rESCs and d-rESCs indicate that even in the same culture environments, rat ESCs still remain in a metastable and heterogeneous state.

Recently, it has been reported that canine ESCs also maintain two kinds of colonies with both domed and flattened morphologies when cultured in complete DMEM/ F-12 supplemented with $15 \%$ fetal bovine serum, indicating that pluripotent cells from other species might also be heterogeneous [9]. Understanding the molecular bases of pluripotent states and the earliest differentiation processes is a fundamental goal in stem cell research. Thus, studying the differences between d-rESCs and f-rESCs could facilitate a better understanding of the origins and molecular characteristics of pluripotent stem cells in different species.

\section{Acknowledgments}

This research was supported by the National Basic Research Program of China (973 Program 2011CBA01000), the 111 Project and the Chinese Science and Technology Key Project (2009ZX10004-403) to H Deng. We express appreciation to Casper Shyr and Pamela A Hoodless (Terry Fox Laboratory, BC Cancer Agency) for their help in microarray data analysis. We are grateful to Jiefang You, Yuhua Han, Lei Tan and other colleagues in our laboratory for technical assistance and advice during experiments. We thank Prof Iain C Bruce of Zhejiang University School of Medicine for his critical revision of this paper.

Yan Shen ${ }^{1, *}$, Cheng $\mathrm{Shi}^{2,}{ }^{*}$, Wei Wei ${ }^{1,3, *}$, Weidong $\mathrm{Yu}^{4}$, Wenlin $\mathrm{Li}^{5}$, Yang Yang ${ }^{1,3}$, Jun $\mathrm{Xu}^{1}$, Wenqin Ying ${ }^{6}$, Xin Sui ${ }^{1,3}$, Lingling Fang ${ }^{6}$, Weiwei Lin ${ }^{6}$, Huan Yang ${ }^{1,3}$, Sheng Ding ${ }^{5}$, Huan Shen ${ }^{2}$, Yan Shi ${ }^{1,3}$, Hongkui Deng ${ }^{1,3}$

${ }^{I}$ Department of Cell Biology and Genetics, College of Life Sciences, Peking University, Beijing 100871, China; ${ }^{2}$ Reproductive Medical Center, Peking University People's Hospital, Peking University, Beijing 100044, China; ${ }^{3}$ Laboratory of Chemical Genomics, School of Chemical Biology and Biotechnology, Shenzhen Graduate School of Peking University, Shenzhen 518055, China; ${ }^{4}$ Institute of Clinical Molecular Biology, Peking University People's Hospital, Peking University, Beijing 100044, China; ${ }^{5}$ Department of Chemistry and the Skaggs Institute for Chemical Biology, The Scripps Research Institute, 10550 North Torrey Pines Road, La Jolla, CA 92037, USA; ${ }^{6}$ Beijing Vital River Laboratory Animal, Inc., Beijing 100012, China

*These three authors contributed equally to this work.

Correspondence: Huan Shen ${ }^{\mathrm{a}}$, Yan Shi ${ }^{\mathrm{b}}$, Hongkui Deng ${ }^{\mathrm{c}}$

${ }^{\mathrm{a}}$ Tel: +86-10-88324439; Fax: +86-10-88324870

E-mail:rmivf@sina.com

${ }^{\mathrm{b}}$ Tel: +86-755-26033257; Fax: +86-755-26033257

E-mail: shiyan@szpku.edu.cn

${ }^{c}$ Tel: +86-10-6275-6954; Fax: +86-10-6275-6954

E-mail: hongkui_deng@pku.edu.cn 


\section{References}

1 Evans MJ, Kaufman MH. Establishment in culture of pluripotential cells from mouse embryos. Nature 1981; 292:154-156.

2 Martin GR. Isolation of a pluripotent cell line from early mouse embryos cultured in medium conditioned by teratocarcinoma stem cells. Proc Natl Acad Sci USA 1981; 78:76347638.

3 Smith AG, Heath JK, Donaldson DD, et al. Inhibition of pluripotential embryonic stem cell differentiation by purified polypeptides. Nature 1988; 336:688-690.

4 Williams RL, Hilton DJ, Pease S, et al. Myeloid leukaemia inhibitory factor maintains the developmental potential of embryonic stem cells. Nature 1988; 336:684-687.

5 Ying QL, Nichols J, Chambers I, et al. BMP induction of Id proteins suppresses differentiation and sustains embryonic stem cell self-renewal in collaboration with STAT3. Cell 2003; 115:281-292.

6 Ying QL, Wray J, Nichols J, et al. The ground state of embryonic stem cell self-renewal. Nature 2008; 453:519-523.

7 Buehr M, Meek S, Blair K, et al. Capture of authentic embryonic stem cells from rat blastocysts. Cell 2008; 135:12871298.

8 Li P, Tong C, Mehrian-Shai R, et al. Germline competent embryonic stem cells derived from rat blastocysts. Cell 2008; 135:1299-1310.

9 Vaags AK, Rosic-Kablar S, Gartley CJ, et al. Derivation and characterization of canine embryonic stem cell lines with in vitro and in vivo differentiation potential. Stem Cells 2009; 27:329-340.

(Supplementary information is linked to the online version of the paper on the Cell Research website.) 